\title{
Comparative efficacy and safety of immunotherapy in the first-line treatment of metastatic renal cell carcinoma: a systematic review and network meta-analysis
}

\author{
Zheran Liu ${ }^{1 \#}$, Ye Chen ${ }^{2 \#}$, Zhigong Wei ${ }^{1 \#}$, Yan $\mathrm{He}^{1}$, Jingjing Wang ${ }^{1}$, Xiaoli $\mathrm{Mu}^{1}$, Ling He ${ }^{1}$, Ruidan $\mathrm{Li}^{1}$, \\ Xiaolin $\mathrm{Hu}^{3}$, Xingchen Peng ${ }^{1}$ \\ ${ }^{1}$ Department of Biotherapy, Cancer Center, West China Hospital, Sichuan University, Chengdu, China; ${ }^{2}$ Department of Medical Oncology, \\ Cancer Center, the State Key Laboratory of Biotherapy, West China Hospital, West China Medical School, Sichuan University, Chengdu, China; \\ ${ }^{3}$ Department of Nursing, West China Hospital, Sichuan University, Chengdu, China \\ Contributions: (I) Conception and design: Z Liu, Y Chen; (II) Administrative support: X Peng, X Hu; (III) Provision of study materials or patients: \\ Z Wei; (IV) Collection and assembly of data: Y He, J Wang, X Mu, L He, R LI; (V) Data analysis and interpretation: Z Liu, Y Chen, Z Wei; (VI) \\ Manuscript writing: All authors. (VII) Final approval of manuscript: All authors. \\ \#These authors contributed equally to this work. \\ Correspondence to: Dr. Xingchen Peng. Department of Biotherpy, Cancer Center, West China Hospital, Sichuan University, No. 37 Guo Xue Alley, \\ Chengdu 610041, China. Email: pxx2014@scu.edu. Dr. Xiaolin Hu. Department of Nursing, West China Hospital, Sichuan University, No. 37 Guo \\ Xue Alley, Chengdu 610041, China. Email: huxiaolin1220@126.com.
}

Backgroundk With the advances in immune checkpoint inhibitor therapy, several novel treatment options for metastatic renal cell carcinoma (mRCC) patients have recently emerged. The present study explored the optimal first-line immunotherapy for mRCC through a Bayesian network meta-analysis of the latest research data.

Methods: PubMed, MEDLINE, EMBASE, American Society of Clinical Oncology (ASCO) meeting abstracts, and the Cochrane Library were searched up to July 2020 to identify any randomized controlled trials related to immunotherapy in the first-line treatment of mRCC. The primary outcome was progressionfree survival, and the secondary outcomes were overall survival and grade 3-4 adverse events.

Results: The network meta-analysis included 4,049 patients from 5 randomized controlled trials. Avelumab plus axitinib and pembrolizumab plus axitinib were the best treatment options in terms of progression-free survival. For overall survival, pembrolizumab plus axitinib had a $77.89 \%$ probability of being the preferred treatment. For adverse events, there was an $89.21 \%$ probability that pembrolizumab plus axitinib was the regimen with the worst side effects.

Conclusions: Through a meta-analysis of the latest available first-line immunotherapy progression-free survival and overall survival data for mRCC, this study found that pembrolizumab plus axitinib might be the best immunotherapy option for first-line treatment. However, attention should be paid to the potential adverse events of this regimen.

Keywords: Metastatic renal cell carcinoma (mRCC); immunotherapy; prognosis; meta-analysis

Submitted Sep 20, 2020. Accepted for publication Dec 14, 2020.

doi: 10.21037/apm-20-1884

View this article at: http://dx.doi.org/10.21037/apm-20-1884 


\section{Introduction}

Renal cell carcinoma (RCC) is one of the deadliest malignancies of the urological system. Each year, there are over 400,000 newly diagnosed cases of RCC and 175,000 related deaths (1). The detection rates of RCC have steadily increased over the past decade due to the incidental diagnosis of renal masses on imaging (2). In $25-30 \%$ of RCC cases, metastatic renal cell carcinoma (mRCC) is detected at initial diagnosis, and the 5-year survival rate of these patients is a meager $12 \%(3-5)$.

Treatment options for mRCC patients have traditionally been limited. Cytokine-based treatments, including agents such as interferon-alpha or interleukin-2 in the 1990s, have achieved modest efficacy (6). However, in recent years, the management and treatment outcomes of mRCC have improved due to increased understanding of the disease's biological mechanism and the emergence of novel treatment options (7). Vascular endothelial growth factor inhibitor therapy has been shown to significantly improve response rates and prolong progression-free survival (PFS) (8). Recently, the emergence of immune checkpoint inhibitor therapy, which can target either programmed cell death protein 1 /programmed death-ligand $1(\mathrm{PD}-1 / \mathrm{PD}-\mathrm{L} 1)$ or cytotoxic T-lymphocyte-associated protein 4 (CTLA-4), has brought hope to mRCC patients (9). Several randomized controlled trials (RCTs) have shown immunotherapy to have PFS and overall survival (OS) benefits over sunitinib for patients with mRCC (10).

The CheckMate 214 trial explored the efficacy of ipilimumab plus nivolumab for mRCC patients. The results showed that this treatment achieved a significantly higher objective response rate $(42 \%$ vs. $27 \%, \mathrm{P}<0.001)$ and OS rate $(75 \%$ vs. $60 \%, \mathrm{P}<0.001)$ than sunitinib (11). The KEYNOTE-426 trial also demonstrated that patients who received pembrolizumab plus axitinib had prolonged 12-month OS (89.9\% vs. 78.3\%) and median PFS (15.1 vs. 11.1 months), as well as an improved objective response rate (58.3\% vs. 35.7\%), when compared to the sunitinib group (12). In 2019, the JAVELIN Renal 101 trial compared the efficacy and safety of avelumab plus axitinib with that of sunitinib. The results showed that compared to that in the sunitinib group, the PFS was significantly prolonged in the avelumab plus axitinib group, with comparable adverse effects (13).

Previous studies have shown the benefits of using immunotherapy as first-line treatment and have described the treatment potential of anti-angiogenesis agents combined with immunotherapy for mRCC. However, a systematic comparison of the effects of different immunotherapy-based regimens on patient survival is still lacking. The present study is the first systematic, mixed treatment comparison including RCTs to compare the outcomes and safety of different immunotherapy-based treatments in the first-line therapy of mRCC. We present the following article in accordance with the PRISMA reporting checklist (available at http://dx.doi.org/10.21037/ apm-20-1884).

\section{Methods}

\section{Literature search strategy}

PubMed, MEDLINE, EMBASE, the American Society of Clinical Oncology (ASCO) meeting abstracts, and the Cochrane Library were searched up to July 2020. The searches were performed using the following terms: "renal cell carcinoma", "first-line treatment or primary therapy", "immunotherapy", and "survival or prognosis", as well as related or extended terms including MESH terms. Only RCTs were included. Reference lists and relevant materials were carefully searched manually. No registration protocol existed or was published previously for the present study.

\section{Inclusion and exclusion criteria}

Studies were included according to the following criteria: (I) RCTs relating to first-line treatment of mRCC; (II) compared the outcomes of immunotherapy treatment and standard mRCC treatment.

Studies were excluded based on the following criteria: (I) republished literature; (II) reviews, comments, or letters; (III) placebo-controlled studies or single-arm studies; (IV) PFS, OS, or adverse events (AEs) not reported.

\section{Data extraction, bias assessment, and beterogeneity analysis}

The studies were independently evaluated by 2 co-authors. Any disagreement was resolved through discussion with a third author. The abstracts and full texts of the studies were read, and the following data were extracted: first author's name, publication year, trial phase, sample size of each treatment group, patient characteristics, treatment options, and treatment outcomes including OS, PFS, or grade 3-4 AEs with the $95 \%$ confidence interval (CI). The risk of bias was evaluated according to the Cochrane Handbook 


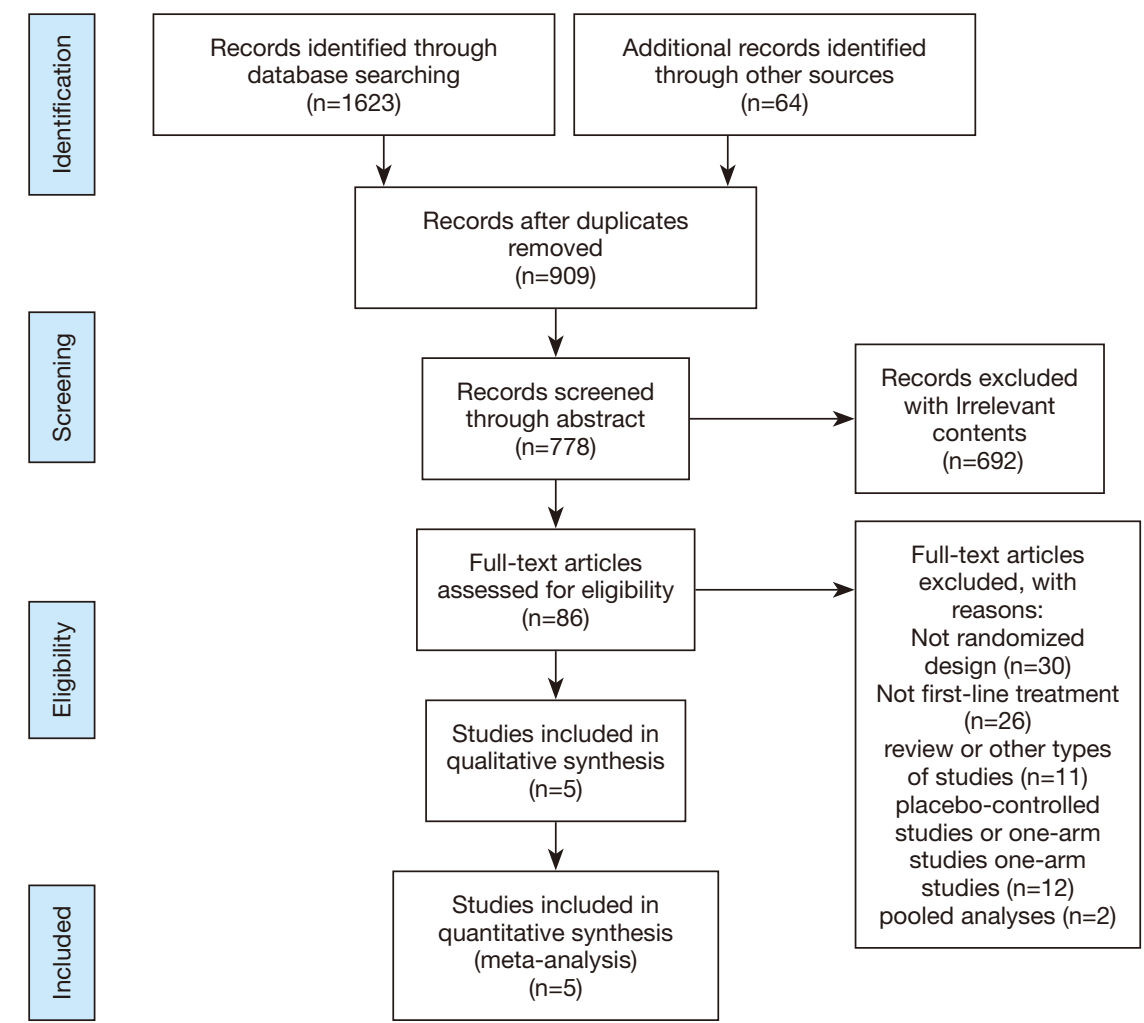

Figure 1 The flow diagram of the literature selection.

for Systematic Reviews of Interventions $(14,15)$. Using the Cochrane tool, we assessed the included studies for potential bias from random sequence generation, allocation concealment, blinding of participants and personnel, blinding of outcome assessment, incomplete outcome data, and selective reporting. Heterogeneity analysis was performed when two or more trials were available for a given comparison. The global 12 was reported for each analysis.

\section{Statistical analysis}

Fixed-effects models with the Bayesian approach were adopted to analyze outcome measurements using the Gemtc package in R 3.6.0 (University of Auckland, New Zealand). For PFS and OS, the network meta-analysis was performed to obtain hazard ratios (HRs) and their $95 \%$ credible intervals (CI) according to a tutorial published by Woods et al. (16). HRs and 95\% CIs were calculated to estimate and compare outcome differences among treatments. For AEs, arm-based analysis was performed using the data from each study. Odds ratios (ORs) and 95\% CIs were used to compare AEs among treatments. Ranking probability were calculated using the Gemtc package (17). Subgroup analyses based on the International Metastatic Renal Cell Carcinoma Database Consortium (IMDC) risk subgroup and PD-L1 positive (PD-L1+) subgroup were conducted. All qualified studies were included without any artificial selection. The surface under the cumulative ranking curve (SUCRA) was used to evaluate the relative ranking and the probability of each treatment being ranked 1 st, 2 nd, etc. for each outcome $(18,19)$.

\section{Results}

\section{Search results}

A total of 1,687 studies were retrieved in the initial search, and the full-texts of 86 studies were reviewed. Eventually, 5 trials involving 4,049 patients were deemed to be eligible for inclusion (11-13,20,21). A detailed flow diagram of the study selection process is presented in Figure 1. PFS data were available for all 4,049 patients in the 5 included trials, while OS data were available for 3,569 patients in 3 
Table 1 The main characteristics of the included studies

\begin{tabular}{|c|c|c|c|c|c|c|c|c|}
\hline Study & Year & Author & Phase & $\begin{array}{l}\text { Experimental arm } \\
\text { (patients number) }\end{array}$ & $\begin{array}{l}\text { Control arm } \\
\text { (patients arm) }\end{array}$ & os & PFS & Grade 3/4 Aes \\
\hline Immoton 150 & 2019 & $\begin{array}{l}\text { David F. } \\
\text { McDermott }\end{array}$ & Phase II & $\begin{array}{l}\text { Atezolizumab + } \\
\text { bevacizumab }(n=101) \\
\text { Atezolizumab }(n=103)\end{array}$ & $\begin{array}{l}\text { Sunitinib } \\
(n=101)\end{array}$ & NA & $\begin{array}{l}\text { HR (Atezolizumab } \\
+ \text { bevacizumab vs. } \\
\text { Sunitinib) }=1.00 \\
(0.69-1.45) ; \mathrm{HR} \\
\text { (Atezolizumab vs. } \\
\text { Sunitinib) }=1.03 \\
(0.82-1.71)\end{array}$ & $\begin{array}{l}\text { Atezolizumab + } \\
\text { bevacizumab (64/101); } \\
\text { Atezolizumab (41/103); } \\
\text { Sunitinib }(69 / 100)\end{array}$ \\
\hline Immotion 151 & 2019 & Brian I Rini & Phase III & $\begin{array}{l}\text { Atezolizumab }+ \\
\text { bevacizumab }(n=454)\end{array}$ & $\begin{array}{l}\text { Sunitinib } \\
(n=461)\end{array}$ & $\begin{array}{l}\mathrm{HR}=0.93 \\
(0.76-1.14)\end{array}$ & $\begin{array}{l}\mathrm{HR}=0.83 \\
(0.70-0.97)\end{array}$ & $\begin{array}{l}\text { Atezolizumab } \\
(182 / 451) ; \text { Sunitinib } \\
(250 / 446)\end{array}$ \\
\hline Checkmate 214 & 2018 & R.J. Motzer & Phase III & $\begin{array}{l}\text { Nivolumab + } \\
\text { Ipilimumab }(n=547)\end{array}$ & $\begin{array}{l}\text { Sunitinib } \\
(n=535)\end{array}$ & $\begin{array}{l}\mathrm{HR}=0.63 \\
(0.44-0.89)\end{array}$ & $\begin{array}{l}\mathrm{HR}=0.82 \\
(0.64-1.05)\end{array}$ & $\begin{array}{l}\text { Nivolumab + } \\
\text { Ipilimumab (250/547); } \\
\text { Sunitinib (335/535) }\end{array}$ \\
\hline KEYNOTE-426 & 2019 & B.I. Rini & Phase III & $\begin{array}{l}\text { Pembrolizumab + } \\
\text { Axitinib }(n=432)\end{array}$ & $\begin{array}{l}\text { Sunitinib } \\
(n=429)\end{array}$ & $\begin{array}{l}H R=0.53 \\
(0.38-0.74)\end{array}$ & $\begin{array}{l}H R=0.69 \\
(0.57-0.84)\end{array}$ & $\begin{array}{l}\text { Pembrolizumab + } \\
\text { Axitinib (325/429); } \\
\text { Sunitinib (300/425) }\end{array}$ \\
\hline
\end{tabular}

AEs, adverse events; NA, not available; OS, overall survival; PFS, progression-free survival; HR, hazard ratio.

trials. Information on grade 3-4 AEs was available for 4010 patients in the 5 trials. The characteristics of each included study are listed in Table 1 (11-13,20,21). The Cochrane Collaboration's tool was used to evaluate the risk of bias for each study. The risk of bias graph and summary are presented in Figure S1. All studies showed a high risk of bias for blinding of participants and personnel. All studies showed a low risk of bias for random sequence generation, blinding of outcome assessment, incomplete outcome data, and selective reporting.

\section{PFS}

A total of 4,049 patients across 5 trials were included in the analysis of PFS $(11-13,20,21)$. The treatment regimens used in the included studies were sunitinib, atezolizumab, atezolizumab plus bevacizumab, nivolumab plus ipilimumab, avelumab plus axitinib, and pembrolizumab plus axitinib. The network comparing with six different treatments was constructed and is shown in Figure $2 A$. The results indicated that avelumab plus axitinib and pembrolizumab plus axitinib were the best regimens for achieving longer PFS in mRCC patients (avelumab plus axitinib: 1st rank probability 39.02\%, compared with sunitinib, HR $=0.69,95 \%$ CI: $0.56-0.85$; pembrolizumab plus axitinib: 1st rank probability $38.60 \%$, $\mathrm{HR}=0.69,95 \%$ CI: $0.57-0.83$, Figure $2 B, C$ ). In this analysis, no significant heterogeneity was found for neither global comparison nor the comparison between atezolizumab plus bevacizumab with sunitinib $\left(\mathrm{I}^{2}=0\right)$.

\section{OS}

A total of 3,569 patients across 3 trials were included in the analysis of OS $(11,12,21)$. The regimens used in the included studies were sunitinib, nivolumab plus ipilimumab, pembrolizumab plus axitinib, and atezolizumab plus bevacizumab. The network was constructed and is shown in Figure 3A. The results showed that pembrolizumab plus axitinib was the best regimen in terms of OS (compared with sunitinib, HR $=0.53$, 95\% CI: $0.38-0.74$, Figure $3 B$ ) and had a $77.89 \%$ probability of being the preferred option for achieving improved OS (Figure 3C). In addition, the OS of the nivolumab plus ipilimumab group was longer than that in the sunitinib group. In this analysis, the global I2 was 0 . 
A

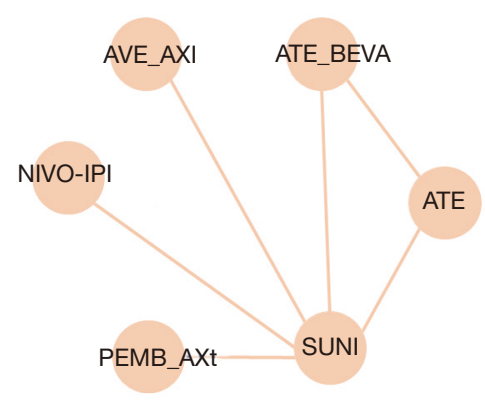

B

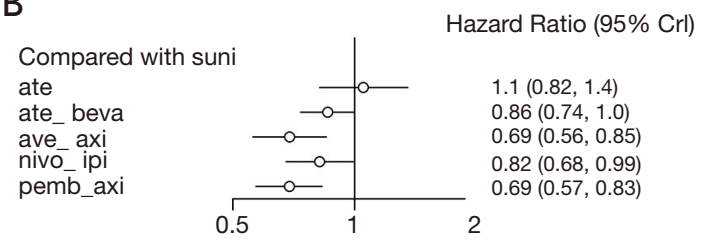

C

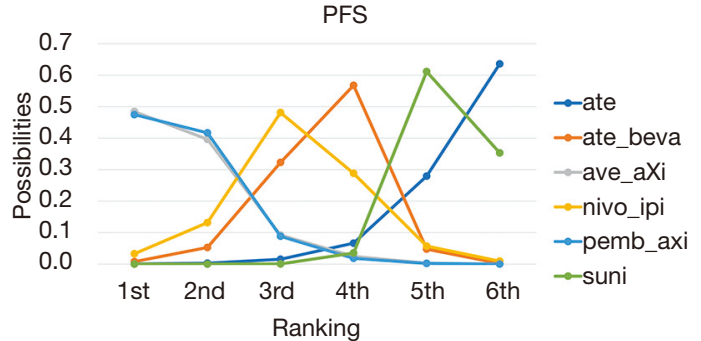

Figure 2 Analysis of progression-free survival: (A) the network diagram; (B) the forest plot of the PFS of all patients, with sunitinib as the comparator (C) the surface under the cumulative ranking curve (SUCRA) plot. For a certain treatment option and a certain outcome, the higher the possibilities (the Y-axis) are, the more likely it is to be the top rank in the SUCRA plot. ave_axi: avelumab plus axitinib; ate: atezolizumab; ate_beva: atezolizumab plus bevacizumab; nivo_ipi: nivolumab plus ipilimumab; pemb_axi: pembrolizumab plus axitinib; suni: sunitinib. SUCRA: the surface under the cumulative ranking curve.

A

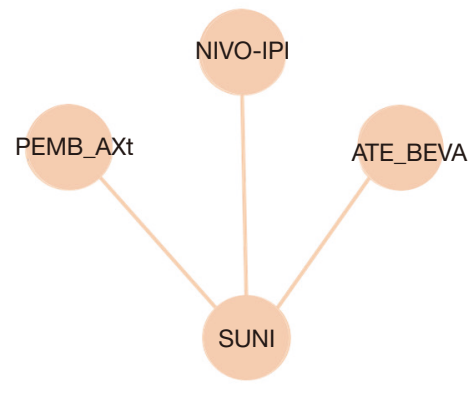

B
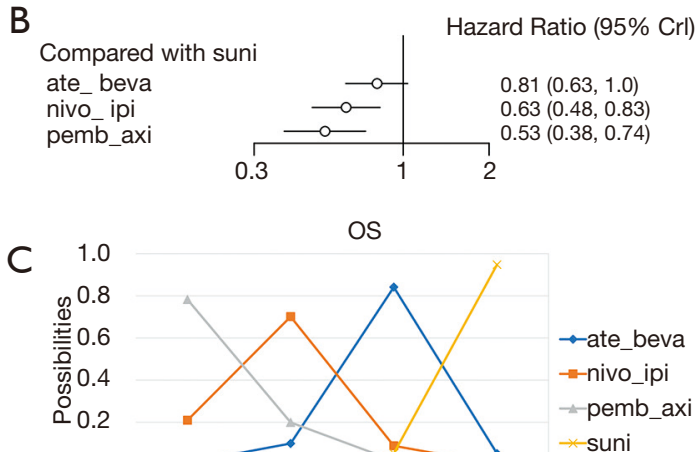

0.0

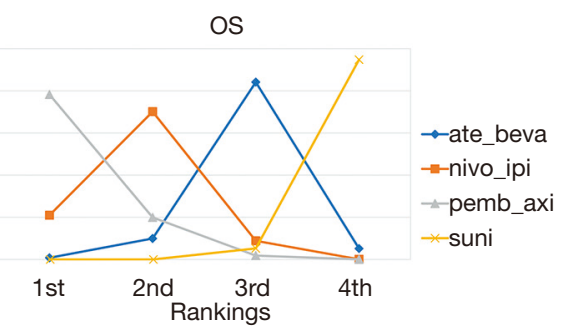

Figure 3 Analysis of overall survival: (A) the network diagram; (B) the forest plot, with sunitinib as the comparator; (C) the surface under the cumulative ranking curve (SUCRA) plot. For a certain treatment option and a certain outcome, the higher the possibilities (the Y-axis) are, the more likely it is to be the top rank in the SUCRA plot. ate_beva: atezolizumab plus bevacizumab; nivo_ipi: nivolumab plus ipilimumab; pemb_axi: pembrolizumab plus axitinib; suni: sunitinib. SUCRA: the surface under the cumulative ranking curve.

\section{AEs}

Five RCTs reported grade 3-4 AEs of 7 treatments (nivolumab plus ipilimumab, atezolizumab plus bevacizumab, pembrolizumab plus axitinib, atezolizumab, avelumab plus axitinib, and sunitinib) in 4,010 patients. The network was constructed and is shown in Figure $4 A$. Atezolizumab alone had less severe AEs compared with sunitinib ( $\mathrm{OR}=0.26,95 \% \mathrm{CI}: 0.15-0.43$, Figure $4 B)$. There was a $99.3 \%$ probability that atezolizumab alone was the best-tolerated regimen, while pembrolizumab plus axitinib had an $89.21 \%$ probability of being the worst-tolerated regimen (Figure 4C). In this analysis, no significant heterogeneity was found for neither global comparison nor the comparison between atezolizumab plus bevacizumab 
A

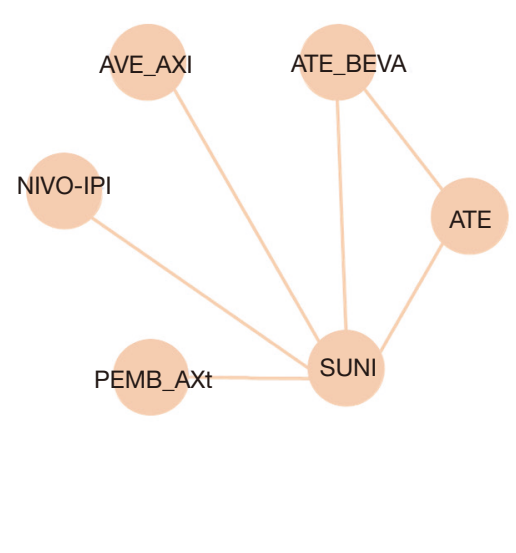

B

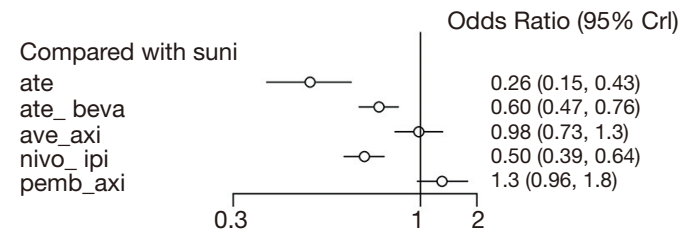

C

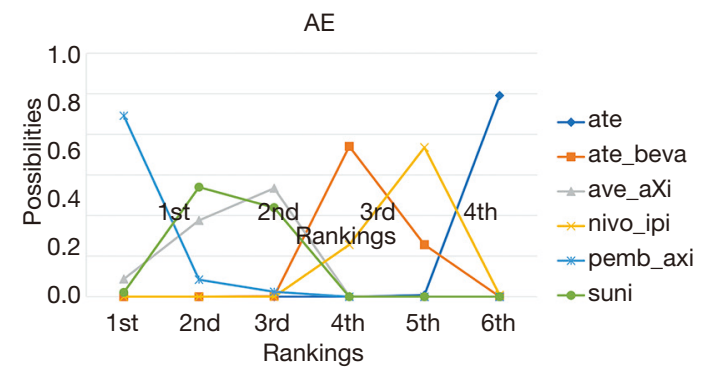

Figure 4 Analysis of adverse effects: (A) the network diagram; (B) the forest plot, with sunitinib as the comparator; (C) the surface under the cumulative ranking curve (SUCRA) plot. For a certain treatment option and a certain outcome, the higher the possibilities (the Y-axis) are, the more likely it is to be the top rank in the SUCRA plot. ave_axi: avelumab plus axitinib; ate: atezolizumab; ate_beva: atezolizumab plus bevacizumab; nivo_ipi: nivolumab plus ipilimumab; pemb_axi: pembrolizumab plus axitinib; suni: sunitinib. SUCRA: the surface under the cumulative ranking curve.

with sunitinib $\left(\mathrm{I}^{2}=0\right)$.

\section{Subgroup analysis}

First, we conducted a network meta-analysis that only included PD-L1+ patients (Table 2, Figure 5A). We found that PD-L1+ patients treated with pembrolizumab plus axitinib had significantly longer PFS than those in the sunitinib group (HR $=0.62$, 95\% CI: 0.47-0.82, Figure 5B). pembrolizumab plus axitinib had an $48.1 \%$ probability of being the preferred option for PD-L1+ patients (Figure 5C). In this subgroup analysis, no significant heterogeneity was found for neither global comparison nor the comparison between atezolizumab plus bevacizumab with sunitinib $\left(\mathrm{I}^{2}=0\right)$.

For further analysis, the patients were also divided into IMDC risk subgroups. We found that for the favorable and intermediate IMDC risk score groups, patients treated with avelumab plus axitinib displayed the best PFS (Figure 6, $\mathrm{HR}=0.50,95 \% \mathrm{CI}: 0.26-0.95$ for the favorable group and $\mathrm{HR}=0.64,95 \% \mathrm{CI}: 0.47-0.87$ for the intermediate group). However, for patients with a poor IMDC risk score, atezolizumab plus bevacizumab had a $42.99 \%$ probability of being the best treatment in terms of PFS (Figure 6, $\mathrm{HR}=0.52,95 \% \mathrm{CI}: 0.22-1.2)$. In this subgroup analysis, the global I ${ }^{2}$ was 0 .

\section{Discussion}

In the past decade, the treatment of mRCC has mainly been centered on the use of anti-angiogenic tyrosine kinase inhibitors such as sunitinib and sorafenib (7). However, recently, mRCC treatment has stepped into a new era with the advances in immunotherapy, especially in the application of immune checkpoint inhibitors, which have become the standard first-line treatment for mRCC (22). In a 2018 meta-analysis, the treatment outcomes of patients who received immunotherapy and the standard treatment for mRCC were compared (23). The results showed that immunotherapy could significantly improve the OS and PFS of mRCC patients (HR $=0.75,95 \%$ CI: $0.66-0.85, \mathrm{P}<0.001$; HR $=0.89,95 \%$ CI: 0.80-0.98, $\mathrm{P}=0.01$, respectively) (24). However, that meta-analysis combined the conclusions of first-line and second-line treatments, making it difficult to determine the effects of first-line treatment alone. Moreover, several RCT results have since been updated, which may affect the validity of the conclusions.

The present study conducted a systematic comparison of first-line immunotherapy treatment options for mRCC, taking into account to several of the latest RCTs. Two phase III RCTs published in 2019 revealed the potential benefits of using immunotherapy as a first-line treatment for mRCC 
Table 2 The main characteristics of patients with PD-L1 positive expression

\begin{tabular}{|c|c|c|c|c|c|}
\hline Study & Phase & $\begin{array}{l}\text { Experimental arm } \\
\text { (patients number) }\end{array}$ & $\begin{array}{l}\text { Control arm } \\
\text { (patients arm) }\end{array}$ & PFS & OS \\
\hline Immotion 150 & Phase II & $\begin{array}{l}\text { Atezolizumab + bevacizumab } \\
(n=50), \text { Atezolizumab }(n=54)\end{array}$ & Sunitinib $(n=60)$ & $\begin{array}{l}\text { Atezolizumab + bevacizumab: } \\
\mathrm{HR}=0.64(0.38-1.08) \\
\text { Atezolizumab: } \\
\mathrm{HR}=1.03(0.63-1.67)\end{array}$ & NA \\
\hline Checkmate 214 & Phase III & Nivolumab + Ipilimumab $(\mathrm{n}=284)$ & Sunitinib $(n=278)$ & NA & $\mathrm{HR}=0.45(0.29-0.71)$ \\
\hline JAVELIN Renal 101 & Phase III & Avelumab + Axitinib $(n=270)$ & Sunitinib $(n=290)$ & $\mathrm{HR}=0.61(0.47-0.79)$ & NA \\
\hline KEYNOTE-426 & Phase III & $\begin{array}{l}\text { Pembrolizumab + Axitinib } \\
(n=243)\end{array}$ & Sunitinib $(n=254)$ & $\mathrm{HR}=0.62(0.47-0.80)$ & $\mathrm{HR}=0.54(0.35-0.84)$ \\
\hline
\end{tabular}

AEs, adverse events; NA, not available; OS, overall survival; PD-L1, programmed death-ligand 1; PFS, progression-free survival; HR, hazard ratio.
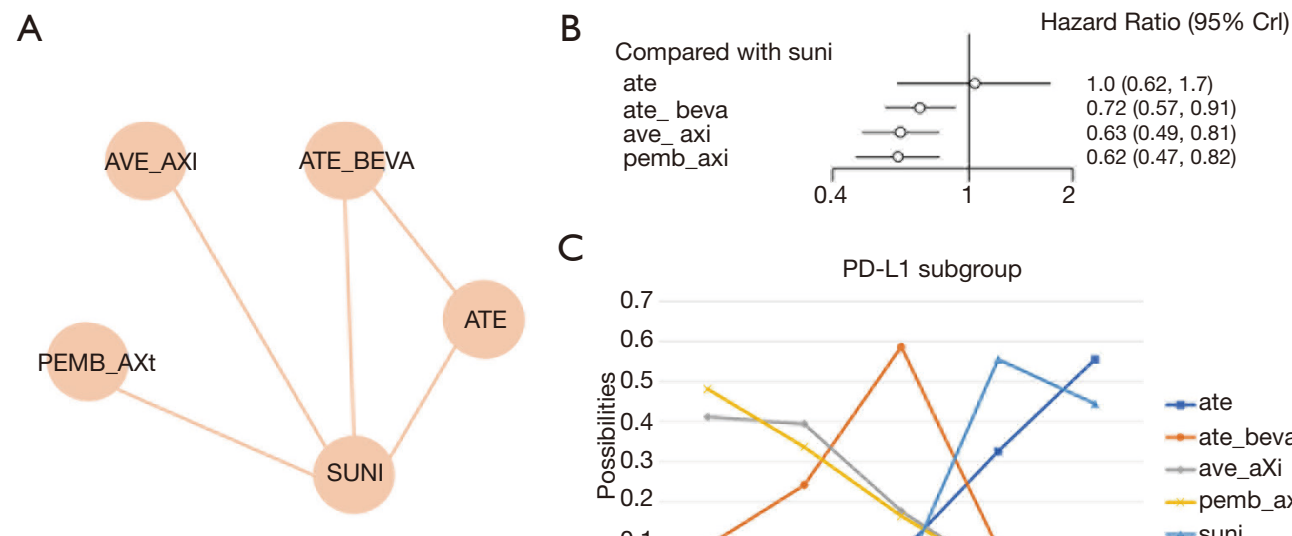

C

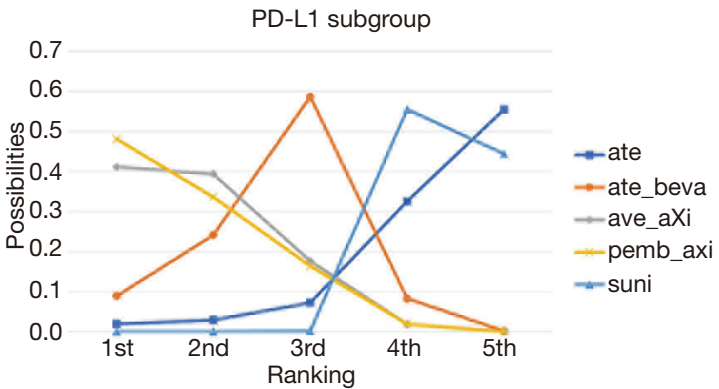

Figure 5 Subgroup analysis of the PFS in the PD-L1 positive patients. (A) the network diagram; (B) the forest plot of the PFS of all patients, with sunitinib as the comparator $(\mathrm{C})$ the surface under the cumulative ranking curve (SUCRA) plot. For a certain treatment option and a certain outcome, the higher the possibilities (the Y-axis) are, the more likely it is to be the top rank in the SUCRA plot. ave_axi: avelumab plus axitinib; ate: atezolizumab; ate_beva: atezolizumab plus bevacizumab; pemb_axi: pembrolizumab plus axitinib; suni: sunitinib. SUCRA: the surface under the cumulative ranking curve.

patients. The phase III JAVELIN Renal 101 trial showed that the median PFS of patients who received avelumab plus axitinib treatment reached 13.8 months, which was significantly longer than the PFS of patients treated with sunitinib (8.4 months). Furthermore, in patients with PDL1+ tumors, treatment using avelumab plus axitinib had a superior objective response rate compared to sunitinib (55.2\% vs. $25.5 \%$ ) (13). The KEYNOTE-426 trial demonstrated that pembrolizumab plus axitinib could significantly prolong the PFS of mRCC patients compared with sunitinib ( $\mathrm{HR}=0.69,95 \% \mathrm{CI}$ : 0.57-0.84, $\mathrm{P}<0.001$ ). Furthermore, the treatment efficacy was present across all subgroups, including IMDC risk groups and patients with and without PD-L1 expression (12). According to the CheckMate 214 trial, nivolumab plus ipilimumab could markedly prolong the OS of patients with PD-L1 expression greater than $1 \%$, while for those with PD-L1 expression levels less than $1 \%$, the OS benefits were not 

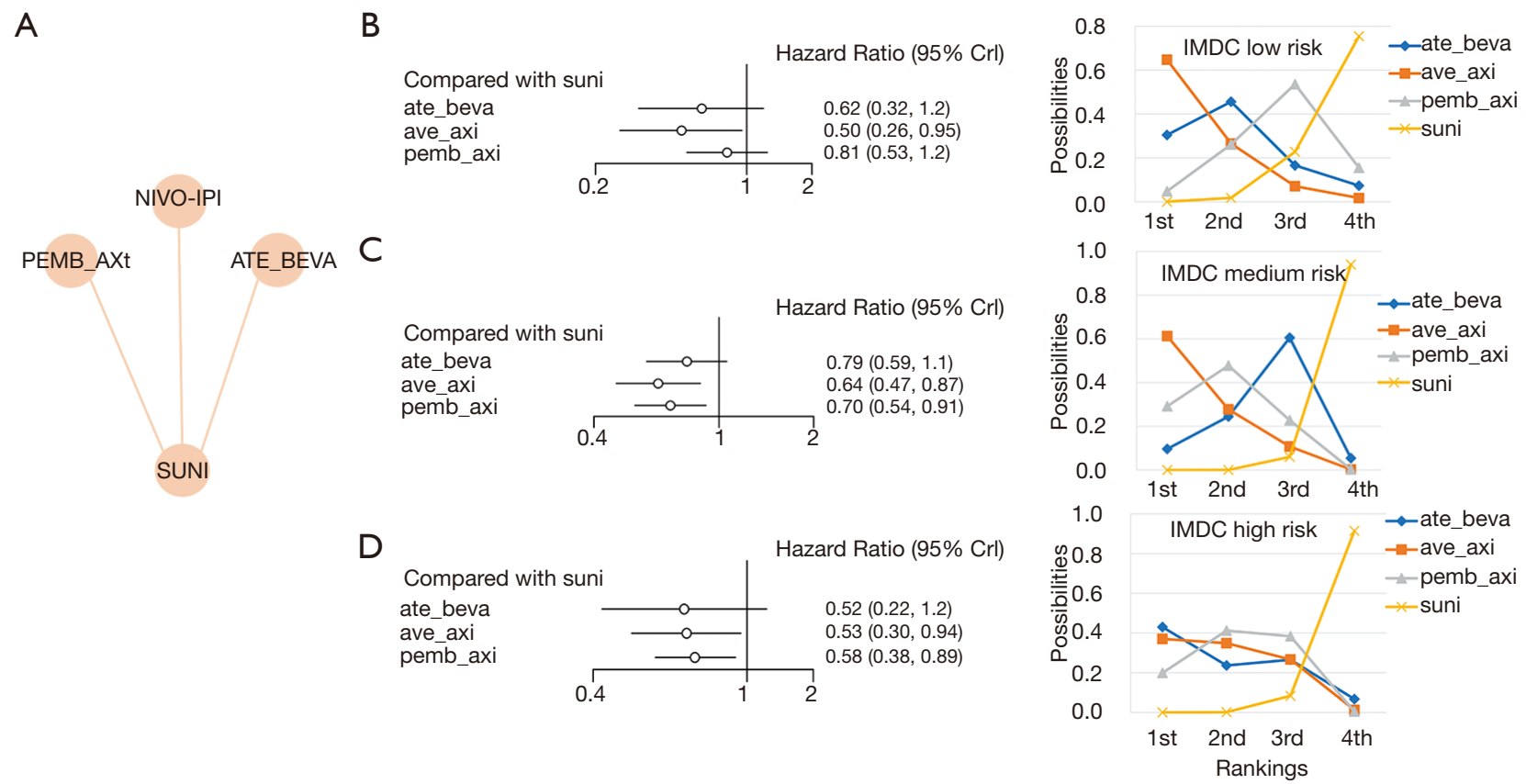

Figure 6 Subgroup analysis of the PFS in the different IMDC risk score. (A) The network diagrams. (B) The forest plot and SUCRA plot for the favorable risk score group. (C) The forest plot and SUCRA plot for the intermediate-risk score group. (D) The forest plot and SUCRA plot for the poor-risk score group. In the SUCRA plot, for a certain treatment option and a certain outcome, the higher the possibilities (the Y-axis) are, the more likely it is to be the top rank. ate_beva: atezolizumab plus bevacizumab; nivo_ipi: nivolumab plus ipilimumab; pemb_axi: pembrolizumab plus axitinib; suni: sunitinib. SUCRA: the surface under the cumulative ranking curve.

significant. Also, nivolumab plus ipilimumab treatment had a much lower risk of grade 3-4 AEs than sunitinib (11).

Previous studies have mostly used sunitinib alone as the control group, despite it long being abandoned as a first-line treatment. As of August 2019, 5 RCTs had compared the efficacy of immunotherapy plus anti-angiogenic therapy with that of sunitinib. Four of them focused on PD-1/L1 inhibitors combined with anti-angiogenic treatment $(12,13,20,21)$, and 1 focused on PD-1 inhibitors combined with a CTLA4 monoclonal antibody (11). Compared with normal metaanalyses, which could only set sunitinib as a global control due to methodological limitations, the present study conducted a network meta-analysis with a Bayesian framework to enable multiple comparisons among each and all treatments (17).

Our analysis ranked the probability of each regimen being the best option for mRCC treatment. Avelumab plus axitinib had a $57.69 \%$ probability of being the most preferred treatment for improving PFS. Pembrolizumab plus axitinib had a $77.89 \%$ probability of being the best option in terms of OS among all first-line treatments. In PD-L1+ patients, pembrolizumab plus axitinib showed a better PFS compared with avelumab plus axitinib. Furthermore, the application of avelumab plus axitinib and pembrolizumab plus axitinib had comparable and acceptable AEs incidence. In general, pembrolizumab plus axitinib might be the best immunotherapy option for first-line mRCC treatment.

The present study has a number of limitations. Firstly, most RCTs described the prognosis and AEs of target drugs compared with sunitinib; therefore, direct comparisons between the outcomes of each immunotherapy agent are still needed. Some studies also lacked OS data, which may have impacted the OS results. Furthermore, the included studies adopted different approaches for risk classification, which limited the effectiveness of the subgroup analyses. Since all the included studies were RCTs, an efficacyeffectiveness gap might have existed, which means the results of the clinical trials cannot be directly disseminated to clinical practice due to a lack of generalizability (25). Lastly, most of the histological types of the patients included in this study were clear cell RCC; hence, the conclusions may not be suitable for application to other histological subtypes. Therefore, more studies are still needed to explore differences in the efficacy of the immunotherapy combinations across different subgroups. 
In conclusion, the combination of immunotherapy and targeted therapy has now become an alternative first-line treatment option for mRCC patients. Based on available firstline immunotherapy PFS and OS data for mRCC, this study demonstrated that pembrolizumab plus axitinib might be the best immunotherapy option for first-line mRCC treatment, although attention should be paid to the $\mathrm{AEs}$ of this regimen.

\section{Acknowledgments}

Funding: The work was supported by National Natural Sciences Foundation of China (81672386 and 81402494 to Peng).

\section{Footnote}

Reporting Checklist: The authors have completed the PRISMA NMA Checklist. Available at http://dx.doi. org/10.21037/apm-20-1884

Conflicts of Interest: All authors have completed the ICMJE uniform disclosure form (available at http://dx.doi. org/10.21037/apm-20-1884). The authors have no conflicts of interest to declare.

Ethical Statement: The authors are accountable for all aspects of the work in ensuring that questions related to the accuracy or integrity of any part of the work are appropriately investigated and resolved.

Open Access Statement: This is an Open Access article distributed in accordance with the Creative Commons Attribution-NonCommercial-NoDerivs 4.0 International License (CC BY-NC-ND 4.0), which permits the noncommercial replication and distribution of the article with the strict proviso that no changes or edits are made and the original work is properly cited (including links to both the formal publication through the relevant DOI and the license). See: https://creativecommons.org/licenses/by-nc-nd/4.0/.

\section{References}

1. Bray F, Ferlay J, Soerjomataram I, et al. Global Cancer Statistics 2018: GLOBOCAN Estimates of Incidence and Mortality Worldwide for 36 Cancers in 185 Countries. CA Cancer J Clin 2018;68:394-424.

2. Wong MCS, Goggins WB, Yip BHK, et al. Incidence and mortality of kidney cancer: Temporal patterns and global trends in 39 countries. Sci Rep 2017;7:15698.
3. Gupta K, Miller JD, Li JZ, et al. Epidemiologic and socioeconomic burden of metastatic renal cell carcinoma (mRCC): A literature review. Cancer Treat Rev 2008;34:193-205.

4. Wiechno P, Kucharz J, Sadowska M, et al. Contemporary treatment of metastatic renal cell carcinoma. Med Oncol 2018;35:156.

5. Zarrabi K, Wu S. Current and Emerging Therapeutic Targets for Metastatic Renal Cell Carcinoma. Curr Oncol Rep 2018;20:41.

6. Klatte T, Rossi SH, Stewart GD. Prognostic factors and prognostic models for renal cell carcinoma: a literature review. World J Urol 2018;36:1943-52.

7. Merza H, Bilusic M. Current Management Strategy for Metastatic Renal Cell Carcinoma and Future Directions. Curr Oncol Rep 2017;19:27.

8. Alsharedi M, Katz H. Check point inhibitors a new era in renal cell carcinoma treatment. Med Oncol 2018;35:85.

9. Barata PC, Rini BI. Treatment of renal cell carcinoma: Current status and future directions. CA Cancer J Clin 2017;67:507-24.

10. Chang AJ, Zhao L, Zhu Z, et al. The past, present and future of immunotherapy for metastatic renal cell carcinoma. Anticancer Res 2019;39:2683-87.

11. Motzer RJ, Tannir NM, McDermott DF, et al. Nivolumab plus Ipilimumab versus Sunitinib in Advanced Renal-Cell Carcinoma. N Engl J Med 2018;378:1277-90.

12. Rini BI, Plimack ER, Stus V, et al. Pembrolizumab plus Axitinib versus Sunitinib for Advanced Renal-Cell Carcinoma. N Engl J Med 2019;380:1116-27.

13. Motzer RJ, Penkov K, Haanen J, et al. Avelumab plus Axitinib versus Sunitinib for Advanced Renal-Cell Carcinoma. N Engl J Med 2019;380:1103-15.

14. Higgins JPT, Altman DG, Gøtzsche PC, et al. The Cochrane Collaboration's tool for assessing risk of bias in randomised trials. BMJ 2011;343:d5928.

15. Higgins JP, Green S. Cochrane Handbook for Systematic Reviews of Interventions Version 5.1.0 [updated March 2011]. Cochrane Collab. 2011.

16. Woods BS, Hawkins N, Scott DA. Network meta-analysis on the log-hazard scale, combining count and hazard ratio statistics accounting for multi-arm trials: A tutorial. BMC Med Res Methodol 2010;10:54.

17. Neupane B, Richer D, Bonner AJ, et al. Network MetaAnalysis Using R : A Review of Currently Available Automated Packages. PLoS One 2014;9:e115065.

18. Mbuagbaw L, Rochwerg B, Jaeschke R, et al. Approaches to interpreting and choosing the best treatments in 
network meta-analyses. Syst Rev 2017;6:79.

19. Antoniou SA, Koelemay M, Antoniou GA, et al. A Practical Guide for Application of Network MetaAnalysis in Evidence Synthesis. Eur J Vasc Endovasc Surg 2019;58:141-4.

20. McDermott DF, Huseni MA, Atkins MB, et al. Clinical activity and molecular correlates of response to atezolizumab alone or in combination with bevacizumab versus sunitinib in renal cell carcinoma. Nat Med 2018;24:749-57.

21. Rini BI, Powles T, Atkins MB, et al. Atezolizumab plus bevacizumab versus sunitinib in patients with previously untreated metastatic renal cell carcinoma (IMmotion151): a multicentre, open-label, phase 3, randomised controlled trial. Lancet 2019;393:2404-15.

Cite this article as: Liu Z, Chen Y, Wei Z, He Y, Wang J, Mu X, He L, Li R, Hu X, Peng X. Comparative efficacy and safety of immunotherapy in the first-line treatment of metastatic renal cell carcinoma: a systematic review and network meta-analysis. Ann Palliat Med 2021;10(3):2805-2814. doi: 10.21037/apm-20-1884
22. Lalani AA, McGregor BA, Albiges L, et al. Systemic Treatment of Metastatic Clear Cell Renal Cell Carcinoma in 2018: Current Paradigms, Use of Immunotherapy, and Future Directions. Eur Urol 2019;75:100-10.

23. Iacovelli R, Ciccarese C, Bria E, et al. Immunotherapy versus standard of care in metastatic renal cell carcinoma. A systematic review and meta-analysis. Cancer Treat Rev 2018;70:112-17.

24. Wallis CJD, Klaassen Z, Bhindi B, et al. First-line Systemic Therapy for Metastatic Renal Cell Carcinoma: A Systematic Review and Network Meta-analysis [Figure presented]. Eur Urol 2018;74:309-21.

25. Nordon C, Karcher H, Groenwold RHH, et al. The "efficacy-effectiveness gap": Historical background and current conceptualization. Value Health 2016;19:75-81. 

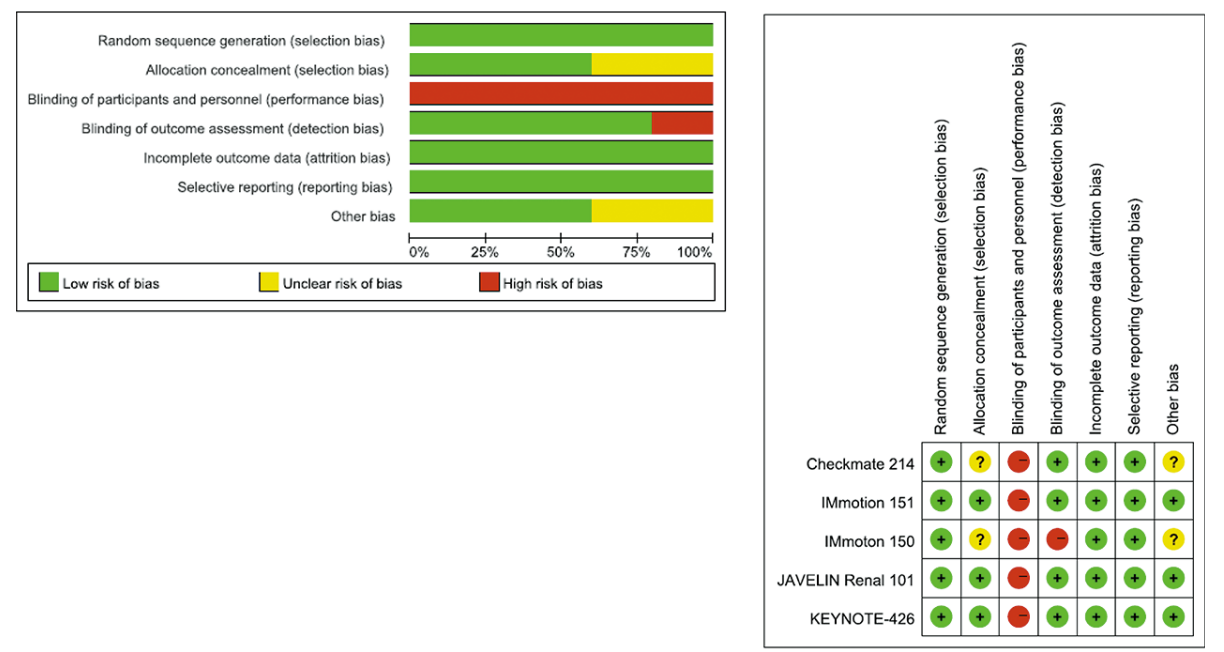

Figure S1 Risk of bias assessment of each included study. 